\title{
Visual asymmetry biases assessment of conjugate lateral eye movement
}

\author{
A. HARVEY BAKER \\ Queens College of the City University of New York, Flushing, New York
}

\begin{abstract}
This paper reports a serendipitous observation. Prior research led to the expectation that equal numbers of right-movers and left-movers would be found in a study aimed at exploring individual differences in conjugate lateral eye movement. Instead, most subjects were found to be right-movers $(p<.001)$. Scrutiny of the study's procedures revealed that the subjects had inadvertently been placed next to the left wall of the room. Apparently asymmetrical visual stimulation during the assessment of conjugate lateral eye movement can affect the right versus left directionality of such movement, an interpretation consistent with earlier formulations and findings of the sensorytonic theory of perception.
\end{abstract}

This paper reports a serendipitous observation that seems to indicate that asymmetrical positioning of a subject in a testing room during assessment of conjugate lateral eye movement (CLEM) biases the right versus left directionality of CLEM. To put this issue into appropriate perspective, the CLEM phenomenon is described and some earlier CLEM findings reviewed. Then, after the report of the study, the implications and interpretation of this finding are considered.

If two people are facing each other while sharing eye gaze and if the first asks the second a question that requires some reflective thinking, the second person will typically avert his/her eyes to the right or left before beginning to reply. This shift in eye gaze has been called conjugate lateral eye movement.

Based on clinical observations, Day (1964) was the first to call attention to CLEM. He reported that most people can be described as either "right-movers" (i.e., a person who shows mostly right CLEMs) or "left-movers." This description turned out to be somewhat oversimplified: it implies a bimodal distribution of CLEM scores, whereas in fact the distribution is relatively flat (cf. Ehrlichman \& Weinberger, 1978, Figure 1). This flat distribution indicates that although many people show predominantly right CLEM and many others show predominantly left CLEM, there are a number who fall in the middle, showing more nearly equal amounts of right and left CLEM. In terms of the issues involved in the present paper, it should be added, however, that if one categorizes a number of people into two groups-those who show more right CLEM versus those who show more left CLEM-the two groups should be approximately equal in size.

Requests for reprints should be sent to A. Harvey Baker, Department of Psychology, NSB E318, Queens College of the City University of New York, Kissena Blvd., Flushing, NY 11367.
In the past two decades, two major lines of CLEM research have emerged. The first has focused on individual differences, with a number of perceptual style and personality correlates of left-mover versus right-mover status having been reported. For example, more dreams are recalled by left-movers (e.g., LeBoeuf, McKay, \& Clarke, 1983-1984), with this effect being greater for males than for females (Van Nuys, 1984). Left-movers attend more to inner experience and feelings (Meskin \& Singer, 1974). Male left-movers are better at a task involving detection of their own heartbeats (Hantas, Katkin, \& Reed, 1984). Male left-movers are more susceptible to hypnosis (Bakan, 1969; Bakan \& Svorad, 1969; R. C. Gur \& R. E. Gur, 1974; Morgan, McDonald, \& MacDonald, 1971).

The second major line of research was inspired by the hypothesis that the left versus right directionality of CLEM reflects greater activation of the contralateral cerebral hemisphere (e.g., Bakan, 1969; Kinsbourne, 1972; Kocel, Galin, Ornstein, \& Merrin, 1972). During the past decade, CLEM thus emerged as one of the main indices of hemisphericity (e.g., Bakan, 1969; Breitling \& Bonnet, 1985; Cole \& Bakan, 1986; Griffiths \& Woodman, 1985; Lenhart \& Katkin, 1986), although certain writers (e.g., Beaumont, 1983; Beaumont, Young, \& McManus, 1984; Ehrlichman \& Weinberger, 1978) have criticized such usage. A number of studies, usually using right-handed subjects, claim that CLEM predominates to the right when the content of the questions is verbal and to the left when the content is spatial (e.g., R. E. Gur, R. C. Gur, \& Harris, 1975; Kinsbourne, 1972; Kocel et al., 1972; Schwartz, Davidson, \& Maer, 1975). With neutral questions, individual differences are pronounced and neither right nor left CLEM predominates (e.g., Duke, 1968).

The present study sought to explore individual differences in right-mover versus left-mover status. Thus, neutral questions were employed with the expectation that approximately equal numbers of right- and left-movers would be identified. 


\section{METHOD}

Subjects

The subjects were 102 right-handed volunteers of college age who were run individually.

\section{Procedure}

The subject sat facing the experimenter, with a table in between them. The experimenter orally administered the questions. After each question, the experimenter recorded the initial direction of each CLEM, as follows: right, left, up, down, diagonal (e.g., up and right, in which case both up and right were recorded), no motion, gaze not shared at the end of the question, or uncertain. In the few instances when a subject's head was not facing completely forward (i.e., toward the experimenter), the data were excluded to prevent any contamination because of head movements.

Each subject was asked 10 questions, patterned after those used by Duke (1968). Based on the CLEM data, each subject was classified as a right-mover (when the amount of CLEM to the right was greater than the amount of CLEM to the left) or as a left-mover (when left CLEM was greater than right CLEM).

\section{RESULTS}

Earlier findings (e.g., Duke, 1968; Ehrlichman \& Weinberger, 1978, especially their Figure 1) indicated that one might expect an equal frequency of right-movers and left-movers. In fact, there were 68 right-movers and 28 left-movers $(p<.0001$, two-tailed binomial test), with the remaining 6 subjects showing equal amounts of right and left CLEM.

\section{DISCUSSION}

Contrary to the expectation based on prior research, we observed a predominance of right-movers. Why? Careful scrutiny of our procedure revealed only one plausible source of this outcome: subjects were inadvertently placed asymmetrically within the room. (The subject's medial axis was $89 \mathrm{~cm}$ from the left wall of the room and $318 \mathrm{~cm}$ from the right wall.) It would appear that the asymmetrical visual stimulation provided by being positioned asymmetrically within the testing room affected the right versus left directionality of CLEM. More specifically, being seated next to the left wall, with most of the visible room extending to the subject's right, resulted in a predominance of rightward CLEM; thus, CLEM predominated toward the center of the wall that the subject faced and saw. Since this study included neither a condition in which the subject sat next to the right wall nor a control condition in which subject sat in the exact center of the room, the present study must be regarded more as a naturalistic than an experimental investigation, and thus the present finding must be viewed as tentative in nature.

Certain earlier CLEM studies touched upon possible effects of asymmetrical stimulation. When Rodin and Singer (1976) varied whether their confederate was seated on the right or left of the experimenter, they found that this variable affected the direction of CLEM for obese subjects, but not for normally weighted subjects, a finding consistent with Schwartz et al.'s (1975) failure to find any effect for this variable with normal subjects. Meskin and Singer (1974) varied location of a painting (to the right or left of the experimenter) and found no effect on the direction of CLEM. Libby and Yaklevich (1973) assessed CLEM in a room with a door to the left of the subject. They found more left than right CLEM. Since their study included no condition with a door to the right, these results must be considered tentative. These earlier studies thus have provided little evidence that environmental asymmetry affects the direction of CLEM.

This lack of earlier evidence of effects of asymmetry does not necessarily contradict our interpretation of the present finding. None of these earlier studies explored the exact type or degree of extreme asymmetry. The failure of these earlier studies to find effects for the types of asym- metry that they explored provides no logical basis for asserting that other forms (or degrees) of asymmetry do not affect the directionality of CLEM. Only future research can clarify this question.

Pending a more definitive study, CLEM researchers would seem well advised to eschew room asymmetry in their studies. Attainment of such a symmetrical setting is something easily done in laboratory studies, but may prove more difficult to achieve by those who choose to explore CLEM in naturalistic settings or with other species (e.g., Muncer, 1982).

We tentatively interpret the present finding that asymmetrical visual stimulation affects the direction of CLEM as being consistent with the earlier formulations and findings of the sensory-tonic theory of perception (e.g., Werner \& Wapner, 1949, 1952). This theory has taken, as one of its central tasks, the demonstration that perception cannot be adequately conceptualized in terms of encapsulated sense modalities (e.g., vision, audition). Its paradigmatic experimental situation has involved the study of the effects of various types of asymmetrical stimulation (e.g., body tilt, head tilt, labyrinthian stimulation, asymmetrical fixation of a visually presented object; cf. Werner \& Wapner, 1956) on perceived object location. Although the more recent theoretical extensions (Wapner, Cirillo, \& Baker, 1969) and findings (e.g., Glick \& Wapner, 1966) indicate that such effects are not necessarily invariant independent of other aspects of the experimental situation, as was implied in the earlier sensory-tonic formulations, the total corpus of findings emerging from the theory provides clear evidence that asymmetrical stimulation does affect various perceptual attributes (cf. Howard \& Templeton's, 1966, review).

Although sensory-tonic theory has admittedly taken perceptual experience as the dependent variable in most of its research, the theory clearly leads to the hypothesis that asymmetrical stimulation should have parallel effects on perception and on sensory-motor (i.e., body) action. This implication was recognized and explored by early sensory-tonic researchers (Chandler, 1953), but the then-existing technology made such research difficult, and results from an extension of this early work were ambiguous (Wapner \& Werner, 1957).

There does seem to be a similarity between the present finding regarding the effect of asymmetrical visual stimulation upon the direction of CLEM and earlier sensory-tonic findings. Sensory-tonic researchers have reported (e.g., Wapner \& Werner, 1957) that when a visual stimulus extended to one side (right or left) of the point of fixation, the apparent straight ahead shifted toward the side to which the visual stimulus extended. Now, in the present study, when the subject sat next to one wall of a room and eye gaze was shared during the question, most of the visible room extended to one side of the subject. The finding here is that CLEM shifted, in parallel fashion, toward the side to which the visible room extended.

\section{REFERENCES}

BAKan, P. (1969). Hypnotizability, laterality of eye movement and functional brain asymmetry. Perceptual \& Motor Skills, 28, 927-932.

BAKAN, P., \& SvORAD, D. (1969). Resting EEG alpha and asymmetry of reflective lateral eye movements. Nature, 223, 975-976.

Beaumont, J. G. (1983). How many brains for how many minds? Educational Psychology, 3, 213-226.

Beaumont, J. G., Young, A. W., \& McManus, I. C. (1984). Hemisphericity: A critical review. Cognitive Neuropsychology, 1, 191-212.

BREITLING, D., \& BoNNET, K. (1985). Lateralization of galvanic skin response, lateral eye movements and a visual half fields recognition task. International Journal of Clinical Neuropsychology, 7, 140-143.

Chandler, K. A. (1953). The effect of moving and non-moving visual stimuli upon head torsion. Unpublished doctoral dissertation, Clark University, Worcester, MA.

Cole, G., \& BAKAN, P. (1986). Alexithymia, hemisphericity, and conjugate lateral eye movements. Psychotherapy \& Psychosomatics, 44, 139-143.

DAY, M. E. (1964). An eye movement phenomenon relating to attention, thought and anxiety. Perceptual \& Motor Skills, 19, 443-446. DUKE, J. (1968). Lateral eye movement behavior. Journal of General Psychology, 78, 189-195. 
Ehrlichman, H., \& Weinberger, A. (1978). Lateral eye movements and hemispheric asymmetry. Psychological Bulletin, 85, 1080-1101.

Glick, J., \& W APNER, S. (1966). Effect of variation in distance between subject and object on space localization. Perceptual \& Motor Skills, 23, 438.

Griffiths, P., \& Woodman, C. (1985). Conjugate lateral eye movements and cognitive mode: Blindness as a control for visually induced oculomotor effects. Neuropsychologia, 23, 257-262.

Gur, R. C., \& GuR, R. E. (1974). Handedness, sex, and eyedness as moderating variables in the relation between hypnotic susceptibility and functional brain asymmetry. Journal of Abnormal Psychology, 83, 635-643.

GUR, R. E., GUR, R. C., \& HARRIS, L. J. (1975). Cerebral activation, as measured by subjects' lateral eye movements, is influenced by experimenter location. Neuropsychologia, 13, 35-44.

Hantas, M., KatkIn, E., \& ReED, S. (1984). Cerebral lateralization and heartbeat discrimination. Psychophysiology, 21, 274-278.

HowARD, I. P., \& TEMPLETON, W. B. (1966). Human spatial orientation. New York: Wiley.

KINSBOURNE, M. (1972). Eye and head turning indicates cerebral lateralization. Science, 176, 539-541.

Kocel, K., Galin, D., Ornstein, R., \& Merrin, E. L. (1972). Lateral eye movements and cognitive mode. Psychonomic Science, 27, 223-224.

LeBoeuf, A., McKay, P., \& Clarke, K. (1983-1984). Lateral eye movements and dream recall in males: A re-appraisal. Imagination, Cognition \& Personality, 3, 61-68.

Lenhart, R., \& Katkin, E. S. (1986). Psychophysiological evidence for cerebral laterality effects in a high-risk sample of students with subsyndromal bipolar depressive disorder. American Journal of Psychiatry, 143, 602-607.

LibBY, W. L., JR., \& YAKLEVICH, D. (1973). Personality determinants of eye contact and direction of gaze aversion. Journal of Personality \& Social Psychology, 27, 197-206.
MESkIN, B. B., \& SINGER, J. L. (1974). Daydreaming, reflective thought, and laterality of eye movements. Journal of Personality \& Social Psychology, 30, 64-71.

Morgan, A. H., McDonald, P. J., \& MacDonald, H. (1971). Differences in bilateral alpha activity as a function of experimental task, with a note on lateral eye movements and hypnotizability. Neuropsychologia, 9, 459-469.

Muncer, S. J. (1982). Functional asymmetry in the chimpanzee. Perceptual \& Motor Skills, 54, 147-152.

Rodin, J., \& Singer, J. L. (1976). Eye-shifts, thought, and obesity. Journal of Personality, 44, 594-610.

Schwartz, G. E., Davidson, R. J., \& Maer, F. (1975). Right hemisphere lateralization for emotion in the human brain: Interactions with cognition. Science, 190, 286-288.

VAN NUYS, D. W. (1984). Lateral eye movement and dream recall: II. Sex differences and handedness. International Journal of Psychosomatics, 31, 3-7.

WapNer, S., Cirillo, L., \& Baker, A. H. (1969). Sensory-tonic theory: Toward a reformulation. Archivio Di Psicologia, Neurologia E. Psichiatria, XXX, 493-512.

WAPNer, S., \& Werner, H. (1957). Perceptual development. Worcester, MA: Clark University Press.

WERnER, H., \& WAPNER, S. (1949). Sensory-tonic field theory of perception. Journal of Personality, 18, 88-107.

WERNER, H., \& WAPNER, S. (1952). Toward a general theory of perception. Psychological Review, 59, 324-338.

WERNER, H., \& WAPNER, S. (1956). Sensory-tonic field theory of perception: Basic concepts and experiments. Revista di psichologia, $\mathbf{5 0}$, 315-337.

(Manuscript received February 13, 1988.) 